\title{
Rincón científico
}

\section{COMUNICACIONES}

${ }^{1}$ Pablo López-Casanova

${ }^{2}$ Manuel Rodríguez-Palma

${ }^{3}$ María Asunción Herrero-Díaz

${ }^{1}$ Máster en Ciencias de la Salud. Universidad de Alicante. Profesor Asociado. Departamento de Enfermería de la Universidad de Alicante.

${ }^{2}$ Máster en Ciencias de la Salud. Universidad de Alicante.

${ }^{3}$ Estudiante de $4^{\circ}$ de Traducción e Interpretación. Universidad de Alicante.

\section{Correspondencia:}

Pablo López Casanova

Departamento de Salud Elche-Hospital General

Agencia Valenciana de Salud

Camino de la Almazara 11. 03203-Elche (Alicante)

Tfno.: 966616142

Fax: 966616141

E-mail: lopez_pabcas@gva.es
Perfil social de los cuidadores

familiares de pacientes dependientes ingresados en el Hospital General Universitario de Elche

\author{
Social profile of the family caregiver \\ of dependant patients \\ in the University General Hospital \\ of Elche
}

Trabajo realizado en la asignatura de Sociología y Salud. Máster en Ciencias de la Salud. Universidad de Alicante.

\section{RESUMEN}

El objetivo de este estudio es describir el perfil social de los cuidadores familiares en el Hospital General Universitario de Elche y conocer el tipo de necesidades que cubren. Metodología: Investigación de tipo descriptivo y transversal, mediante 32 encuestas realizadas entre enero y febrero de 2008, en el Servicio de Medicina Interna. Se efectuó un análisis descriptivo y las variables fueron edad, sexo, ingresos económicos, vivienda, trabajo, parentesco del cuidador y permanencia en el centro hospitalario. Resultados: El perfil del cuidador informal corresponde a una mujer, con una media de edad de 54 años y que permanece todo el día en el hospital. Las necesidades que el cuidador distingue como más importantes estuvieron relacionadas con la compañía, la vigilancia y las necesidades básicas. Conclusiones: El perfil del cuidador familiar es de cónyuges o madres. La realización de las tareas de cuidador supone un gasto de tiempo, dinero, desgaste físico, psicológico y social. Parte de las necesidades del paciente son cubiertas por los cuidadores informales, que reciben poca información y asesoramiento del personal de enfermería sobre cómo realizarlas. Por tanto, ampliar la mirada enfermera es una exigencia y demanda de nuestra sociedad.

\section{PALABRAS CLAVE}

Cuidador familiar, hospital, perfil social, dependencia.

\section{SUMMARY}

The aim of this study is to describe the social profile of the family caregivers in the University General Hospital of Elche, and to know the sort of needs that they cater for. Methodology: This is a descriptive and transversal piece of research, performed by means of 32 surveys done between January and February 2008 in the Internal Medicine service. A descriptive analysis was carried out; such analysis bore in mind the following variables: the caregivers' age, sex, income, dwelling, employment, their kinship with the patient and the lenght of stay at the hospital. Results: The profile of the average informal caregiver corresponds to a woman of approximately 54 years old who stays all day in the hospital. Caregivers consider that the most important needs they meet are related to keeping their relative company, watching over them, and meeting their basic needs. Conclusions: With respect to the profile of family caregivers, spouses and mothers account for most caregivers. The tasks carried out by caregivers imply spending their time and money, and also physical, pysichological and social exhaustion. Part of the patients' needs are covered by informal caregivers, who receive little information and advising from the nursing staff about how to provide care for their disabled relatives. Therefore, nurses doing a closer monitoring of patients and caregivers and paying more attention to them is a requirement and a demand of our society.

\section{KEY WORDS}

Family caregiver, hospital, social profile, dependence, disability.

\section{INTRODUCCIÓN}

Los cuidadores familiares de personas mayores dependientes son aquellas que, por diferentes motivos, coinciden en la labor a la que dedican gran parte de su tiempo y esfuerzo: permitir que otras personas puedan desenvol- verse en su vida diaria, ayudándolas a adaptarse a las limitaciones que su discapacidad funcional (entendida en sentido amplio) les impone.

Si pensamos en que cada vez es mayor el porcentaje de población de más edad, debido fundamentalmente al au- 


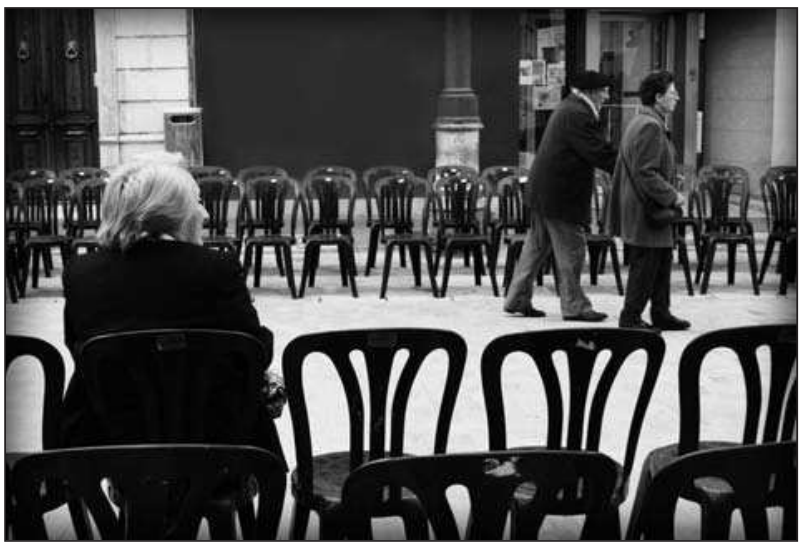

mento de la expectativa de vida y a la disminución de la tasa de natalidad, resulta evidente que aumentará, en un futuro próximo, el número de personas que necesiten algún tipo de ayuda y, por tanto, el número de cuidadores.

Según datos del IMSERSO, en España se estima que el porcentaje de personas mayores que presentan una dependencia importante está entre un $10 \%$ y un $15 \%$ de las personas mayores de 65 años (1). Hay que reconocer la existencia de los problemas comunitarios relativos a las necesidades particulares de las personas mayores y que éstos deben ser afrontados con políticas adecuadas referidas a salud, alimentación, vivienda, familia, medioambiente, bienestar social, seguridad de pensiones y empleo (2).

Los pacientes hospitalizados no están exentos de estos problemas, sino que estas necesidades se ven incrementadas y, por tanto, se produce una situación de estrés tanto en los mayores como en los familiares (3).

Para proporcionar un buen cuidado al enfermo es necesaria la intervención de todos los miembros de la familia, teniendo como único objetivo el cuidado y bienestar del anciano. En la actualidad, la familia es la que asume la mayor parte del cuidado de estas personas. En cada familia suele haber un cuidador principal que responde a las circunstancias de cada familia, sin que se haya llegado a ello por un acuerdo explícito entre los miembros de la familia.

Los estudios sobre cuidadores familiares han sido ampliamente abordados en el ámbito de la Atención Primaria y del entorno doméstico en relación con determinadas enfermedades y patologías (oncología, Alzheimer, ictus, etc.) $(4,5)$, también podemos encontrar estudios (6-9) que inciden sobre su realidad dentro de las instituciones sanitarias en sus diferentes modalidades asistenciales.

La integración del cuidador como parte imprescindible de los cuidados que se prestan a los pacientes dependientes viene determinada principalmente por la estructura de la organización hospitalaria, pero no se deben olvidar los modelos descritos por Twigg: cuidadores como elemento de producción del bienestar, cuidador como recurso, cuidador como cliente y cuidador como co-trabajador (10).

El conocimiento, tanto del modelo de cuidador que tenemos en nuestro entorno como de su perfil social, nos debe ayudar a comprender las necesidades tanto de ellos 201 como de los pacientes que tratamos. Es importante que el cuidador se implique en los cuidados del paciente ya que así se fomenta la relación con el enfermo y se le ayuda a superar el miedo y la idea de no saber actuar con su familiar, pero también es importante que la organización hospitalaria cuente con un análisis del perfil social que nos ayudará a entender a qué población van dirigidos nuestros cuidados y qué podemos esperar de ellos.

El objetivo de este estudio es describir el perfil social de los cuidadores familiares en el Hospital General Universitario de Elche y conocer el tipo de necesidades que cubren.

\section{MATERIAL Y MÉTODOS}

El estudio es de tipo descriptivo y transversal y se realizó mediante encuestas. La recogida de datos se llevó a cabo entre el 15 de enero y el 15 de febrero de 2008 en el Hospital General Universitario de Elche, en el Servicio de Medicina Interna.

Se realizo un muestreo aleatorio simple y se efectuaron 32 encuestas (Anexo). A todos los cuidadores familiares que participaron en el estudio se les pidió su consentimiento solamente verbal y se les informó sobre la confidencialidad de los datos obtenidos. Así mismo se les explicó la naturaleza del estudio.

Los cuidadores familiares fueron seleccionados por el mismo enfermero que realizó el estudio atendiendo a los criterios de inclusión siguientes:

- Más de tres días de permanencia en el hospital.

- Presencia continuada del familiar de, al menos, la jornada matinal y vespertina.

La encuesta se realizó fuera de las habitaciones para controlar el posible sesgo de respuesta por la presencia de otros familiares o del propio paciente.

Para el análisis descriptivo se determinaron las siguientes variables a estudio:

- Edad, sexo, ingresos económicos, convivencia, trabajo, parentesco del cuidador familiar, tiempo de permanencia en el centro hospitalario.

- Relación de actividades que provee a su familiar.

Se utilizo la encuesta de Quero y cols. (6) modificada para los objetivos de este estudio.

\section{RESULTADOS}

Se realizaron 32 encuestas en el Servicio de Medicina Interna del HGU de Elche. La población encuestada se distribuyó entre un $84,4 \%$ de mujeres y un 15,6\% de hombres, con una media de edad de 54,06 $\pm 12,16$ años. En cuanto a la ciudad de procedencia de los cuidadores, más de la mitad $(53,1 \%)$ vivía en Elche, un $25 \%$ en Santa Pola y un $21,9 \%$ en Crevillente.

La distribución según su situación socio-laboral se describe en la Tabla 1.

En cuanto al estatus de empleo, la distribución fue bastante heterogénea en el tipo de contrato y duración. Es ex- 
López-Casanova, P.; Rodríguez-Palma, M.; Herrero-Díaz, Ma.A.: PERFIL SOCIAL DE LOS CUIDADORES FAMILIARES DE PACIENTES DEPENDIENTES INGRESADOS EN EL HOSPITAL GENERAL UNIVERSITARIO DE ELCHE

\begin{tabular}{|l|l|l|}
\hline \multicolumn{3}{|c|}{ Tabla 1. Situación socio-laboral } \\
\hline & Frecuencia & Porcentaje \\
\hline Ama de casa & 15 & 46,9 \\
\hline Jubilado & 6 & 18,8 \\
\hline Trabajo fuera & 11 & 34,4 \\
\hline
\end{tabular}

plicativo por sí sólo el porcentaje de desempleados (Fig.1 y Tabla 2).

Los ingresos que los cuidadores perciben se situaron entre el $40,6 \%$ que no recibía ninguno, el $21,9 \%$ que recibía entre 400 y 600 euros, el 15,6\% entre 1.000 y 1.500 euros y el $12,5 \%$ que recibe entre 600 y 1000 euros. Existió un porcentaje del $3,1 \%$ que recibe menos de 400 euros, al mismo tiempo que hubo un $3,1 \%$ que prefirió no contestar.

En referencia al ámbito en donde viven, el porcentaje mayoritario $(59,4 \%)$ reside en el casco urbano, encontrando una distribución paralela $(31,3 \%$ y $28,1 \%)$ entre casco urbano moderno y antiguo. Un $25 \%$ vive en residencias rurales y un 15,6\% en residencias de ámbito o influencia meramente turística.

La relación entre cuidador y paciente fue mayoritariamente en relación de consanguinidad en primer grado o cónyuge/esposa, situándose en un $93,8 \%$ y encontrándose un 6,3\% de cuidadores que la relación que les unía era política (suegros/nueras).

Los resultados anteriores están estrechamente relacionados con la existencia o no de convivencia en el mismo hogar, hallándose un $65,6 \%$ de cuidadores que convive con los pacientes.

En cuanto al tiempo que dedican a cuidar a los pacientes, podemos verlo en la Tabla 3.

Los días de ingreso y de cuidados se situaron en una media de $7 \pm 2,14$ días de estancia, con un intervalo de 412 días.

Una de las preguntas que se les formularon fue si recibían ayuda en algún momento de algún familiar cercano, a lo que contestaron en un $65,6 \%$ afirmativamente y en un $34,4 \%$ negativamente.

A la pregunta sobre ¿Qué actividades realiza a su paciente?, en la Tabla 4 se describen los porcentajes de respuestas encontradas.

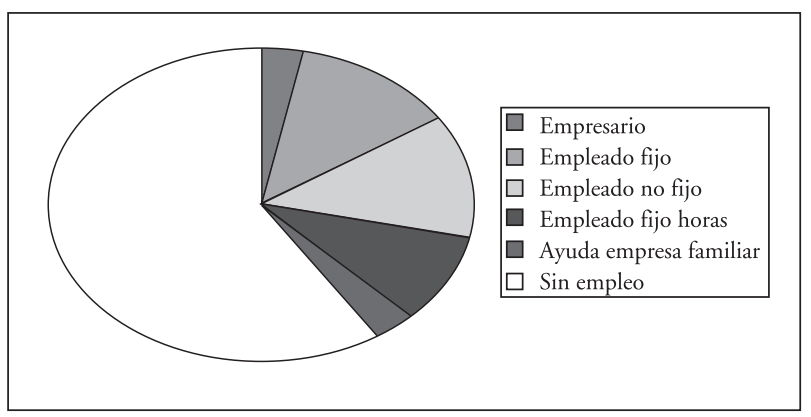

Fig. 1. Ocupación.

\begin{tabular}{|l|l|l|}
\hline \multicolumn{3}{|c|}{ Tabla 2. Estatus de empleo } \\
\hline & Frecuencia & Porcentaje \\
\hline Empresario & 1 & 3,1 \\
\hline Empleado fijo & 4 & 12,5 \\
\hline Empleado no fijo completo & 4 & 12,5 \\
\hline Empleado fijo horas & 3 & 9,4 \\
\hline Ayuda empresa familia & 1 & 3,1 \\
\hline Sin empleo & 19 & 59,4 \\
\hline
\end{tabular}

\begin{tabular}{|l|l|l|}
\hline \multicolumn{3}{|c|}{ Tabla 3. Horas de dedicación } \\
\hline & Frecuencia & Porcentaje \\
\hline Mañana y tarde & 12 & 37,5 \\
\hline Noche & 5 & 15,6 \\
\hline Todo el día & 15 & 46,9 \\
\hline
\end{tabular}

\section{DISCUSIÓN Y CONCLUSIONES}

Como enuncia la Conferencia Española de Organizaciones de Mayores (11), "La dependencia se define como la pérdida de forma temporal o definitiva de la capacidad de la persona para ser autónoma y conlleva un conjunto de dificultades y de sufrimiento para su entorno". La situación social de dependencia es aquella situación personal que requiere la ayuda prolongada de una tercera persona para realizar los actos vitales de la vida cotidiana, es la necesidad de una atención o cuidados prolongados. Y, por tanto, como escribe Rodríguez Cabrero (12), "para proporcionar un buen cuidado al enfermo es necesaria la intervención de todos los miembros de la familia, teniendo como único objetivo el cuidado y bienestar del anciano".

Estos dos posicionamientos son clave para determinar el perfil del cuidador en pacientes dependientes y así poder encontrar puntos de similitud con otros trabajos anteriores y también puntos discordantes. El perfil de dichos cuidadores coincide con los trabajos de Portillo y cols., que describe a una mujer de unos 45-50 años de edad que, a su vez, es ama de casa y lo que las une con sus pacientes son los lazos familiares directos.

Está clara también la relación de desempleo con el cuidado directo a los pacientes dependientes y así mismo relacionado con la cantidad de horas dedicada a su cuidado.

Las actividades que se realizan por parte de los cuidadores coinciden de igual manera con los estudios anteriores, observándose una dedicación exclusiva en el acompañamiento. La vigilancia en la toma de medicación y las necesidades de comer, excreción y lavarse son las que se relacionan como más realizadas. Sin embargo, cuando se pregunta por actividades consideradas de "obligación" profesional, los cuidadores parecen declinar la realización. Tenemos que entender que se han estudiado a cuidadores de 
López-Casanova, P.; Rodríguez-Palma, M.; Herrero-Díaz, Ma.A.: PERFIL SOCIAL DE LOS CUIDADORES FAMILIARES DE PACIENTES DEPENDIENTES INGRESADOS EN EL HOSPITAL GENERAL UNIVERSITARIO DE ELCHE

170

\begin{tabular}{|l|l|l|}
\hline \multicolumn{3}{|c|}{ Tabla 4. } \\
\hline Actividades & Sí & No \\
\hline 1. Peinar, lavar la cara, lavar los dientes & $75 \%$ & $25 \%$ \\
\hline 2. Bañar en la cama & & $100 \%$ \\
\hline 3. Poner la cuña/botella & $81,3 \%$ & $18,8 \%$ \\
\hline 4. Ayudarlo a levantarse & $18,8 \%$ & $81,3 \%$ \\
\hline 5. Acompañar, entretener & $100 \%$ & - \\
\hline 6. Vigilar los sueros & $62,5 \%$ & $37,5 \%$ \\
\hline 7. Moverlo en la cama & $40,65 \%$ & $59,4 \%$ \\
\hline 8. Ducharlo & $12,5 \%$ & $87,5 \%$ \\
\hline 9. Acompañar al cuarto de baño & $6,3 \%$ & $93,8 \%$ \\
\hline 10. Darle la comida & $81,3 \%$ & $18,8 \%$ \\
\hline 11. Ayudarlo a pasear & $9,4 \%$ & $90,6 \%$ \\
\hline 12. Dar medicación o vigilar que la tome & $81,3 \%$ & $18,8 \%$ \\
\hline 13. Dar masajes & $25 \%$ & $75 \%$ \\
\hline
\end{tabular}

necesitado el apoyo de un estudio etnográfico 203 y cualitativo para determinar personalmente con los cuidadores mediante encuestas abiertas unas conclusiones más profundas.

El primer paso de determinar el perfil social básico de los cuidadores se ha concluido y con los datos recogidos podemos afirmar que no parecen existir diferencias de perfil con estudios anteriores más amplios sobre pacientes quirúrgicos y médicos. El perfil puede ser necesario para determinar, como dice Twigg, cuál es el modelo de cuidador en nuestro hospital y poder proveer cuidados también a ellos.

\section{AGRADECIMIENTOS}

Quiero agradecer a la Dirección del HGU de Elche la colaboración prestada en la realización de este estudio y a los cuidadores y compañeros del Servicio de Medicina Interna por haberme prestado su apoyo.

pacientes dependientes y que posiblemente las actividades que requieren fuerza física o la ayuda de otra persona, se obvie su realización por el bien del paciente y cuidador.

Este estudio tiene limitaciones en cuanto que se ha realizado un estudio puramente descriptivo y se hubiera
"Actualmente la filosofía de la calidad se fundamenta en tres pilares básicos: responder a las necesidades del cliente, mejorar continuamente los servicios y crear sistemas apropiados para la mejor producción". Edward Fleming

\section{ANEXO. ENCUESTA SOBRE PERFIL SOCIAL}

\section{Datos del cuidador Sexo: \\ Edad:}

¿Cuál es su situación laboral actual?

1. Ama de casa

2. Jubilado/pensionista

3. Parado

4. Estudiante

5. Trabajando fuera del hogar

\section{Ocupación:}

1. Empresario/a con empleados/as

2. Empresario/a sin empleados/as

3. Miembro cooperativa

4. Empleado/a fijo/a

5. Empleado/a no fijo/a a tiempo total

6. Empleado/a no fijo/a por horas

7. Ayuda en una empresa familiar

¿Qué ingresos se perciben mensualmente en su hogar?
1. Menos de $400 €$
2. De 400 a $600 €$
3. De 600 a $1.000 €$
4. De 1.000 a $1.500 €$
5. De 1.500 a $2.500 €$
6. Más de $2.500 €$
7. NS
8. NC

Localidad del cuidador: ámbito:

¿El paciente vive con $\mathrm{Vd}$.?
1. Sí
2. No

¿Cuál es su relación o parentesco con el paciente? 


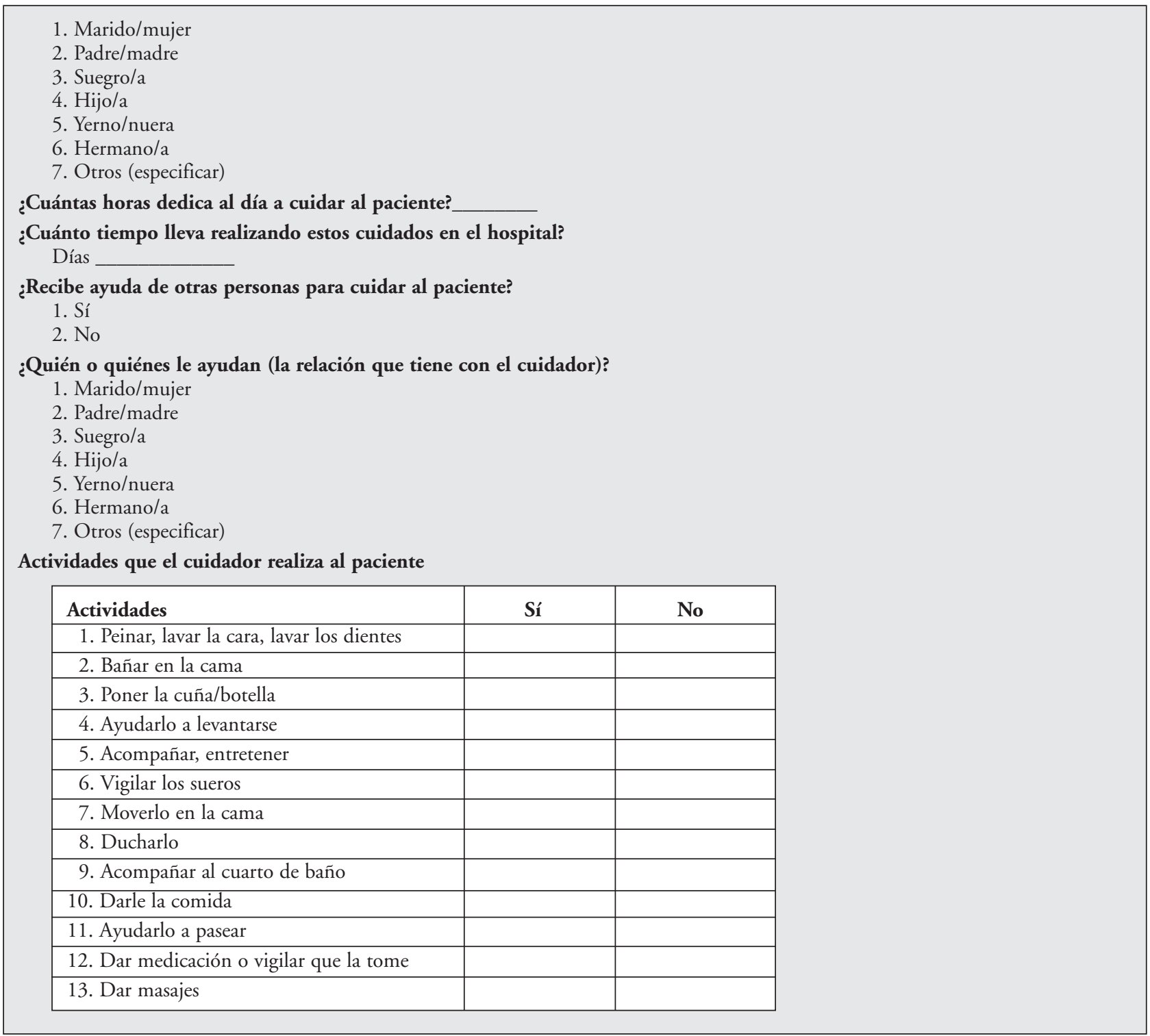

\section{BIBLIOGRAFÍA}

1. IMSERSO. Libro Blanco de Atención a las personas en situación de dependencia en España. Madrid: Ministerio de Trabajo y Asuntos Sociales, 2005.

2. García Castillo N, Pérez Fonollá Ma D. Implantación de un programa de apoyo al cuidador del anciano dependiente hospitalizado. Nure Investigación 2005; 17.

3. Contreras IM, Reyes AP, Marmolejo M, Reyes Ortiz CA. Calidad de vida para los pacientes adultos mayores en las salas de Medicina Interna del Hospital Universitario del Valle: el papel de la recreación. En: II Simposio Nacional de vivencia y gestión en recreación. Cali 22-24 de noviembre de 2001. Colombia: Funlibre, 2001.

4. Portillo MC, Wilson-Barnett J, Saracíbar MI. Estudio desde la percepción de pacientes y familiares del proceso de participación informal en el cuidado después de un ictus: metodología y primeros resultados. Enfermería Clínica 2002; 12 (3): 94-103.

5. Portillo MC, Wilson-Barnett J, Saracíbar MI. Análisis de un marco conceptual para el proceso de participación informal en el cuidado después de un ictus. Enfermería Clínica 2002; 12 (4): 143-51.
6. Quero A, Briones R, Prieto MA, Navarro A, Pascual N, Ruiz C. Perfil y realidad social de los cuidadores familiares en un hospital de traumatología y rehabilitación. Nure Investigación 2004; 9.

7. Quero A. Los cuidados no profesionales en el hospital: la mujer cuidadora. Enfermería Clínica 2003; 13 (6): 348-56.

8. Flores ML, Cano-Caballero MD, Caracuel A et al. La calidad de vida de los acompañantes de pacientes hospitalizados de media y larga estancia. Índex de Enfermería 2002; 38 (11): 18-22.

9. Flores ML, Cano-Caballero MD, Caracuel A et al. La alimentación del acompañante del paciente hospitalizado. Enfermería Clínica 2000; 10 (1): 7-12.

10. Twigg J. Cuidadores de los ancianos: modelos para un análisis. En: Jamieson A. Comparación de políticas europeas de atención a las personas ancianas. Barcelona: SG, 1993. pp. 35-50.

11. Toronjo AM. Cuidador formal frente a cuidador informal. Gerokomos 2001; 12 (2): 59-66.

12. CEOMA. Acompañar y atender la dependencia de tus mayores. Madrid: Ed. Bayard, 2004.

13. Rodríguez Cabrero G. La protección social de la dependencia. Madrid: Ministerio de Asuntos Sociales, 2000. 